\title{
Short genome report of cellulose- producing commensal Escherichia coli 1094
}

\author{
Joaquin Bernal-Bayard ${ }^{1 *}$, Laura Gomez-Valero ${ }^{2,3}$, Aimee Wessel ${ }^{1}$, Varun Khanna ${ }^{4}$, Christiane Bouchier ${ }^{5}$ \\ and Jean-Marc Ghigo ${ }^{1 *}$ id
}

\begin{abstract}
Bacterial surface colonization and biofilm formation often rely on the production of an extracellular polymeric matrix that mediates cell-cell and cell-surface contacts. In Escherichia coli and many Betaproteobacteria and Gammaproteobacteria cellulose is often the main component of the extracellular matrix. Here we report the complete genome sequence of the cellulose producing strain E. coli 1094 and compare it with five other closely related genomes within E. coli phylogenetic group A. We present a comparative analysis of the regions encoding genes responsible for cellulose biosynthesis and discuss the changes that could have led to the loss of this important adaptive advantage in several E. coli strains. Data deposition: The annotated genome sequence has been deposited at the European Nucleotide Archive under the accession number PRJEB21000.
\end{abstract}

Keywords: E. coli, Commensal, Biofilm, Cellulose, Extracellular matrix, Bcs operon

\section{Introduction}

Biofilms are ubiquitous microbial communities growing in close association with surfaces present in natural and anthropic environments [1]. Biofilm bacteria often selfassemble by producing a cohesive extracellular matrix that protects these multicellular aggregates against environmental changes and maintains the integrity of the biofilm structure [2]. Cellulose is a homo-polysaccharide composed of glucose units linked by $\beta-1 \rightarrow 4$ glycosidic bonds and is the most common organic compound on Earth [3]. Initially studied in Gluconacetobacter xylinus, cellulose production is a widespread phenomenon shared by many commensal and pathogenic Betaproteobacteria and Gammaproteobacteria, including Salmonella enterica serovar Typhimurium, Klebsiella pneumoniae, Burkholderia mallei, Shigella boydii, Yersinia enterocolitica, Vibrio fischeri, Pseudomonas putida and many Escherichia coli strains $[4,5]$. Here we report the complete genome sequence of E. coli 1094, a biofilm forming and cellulose-producing strain isolated from the feces of a healthy human male. E. coli 1094 lacks virulence factors commonly associated with pathogenic E. coli and is a member of E. coli phylogenetic group A [6, 7].

\footnotetext{
*Correspondence: joaquin.bernal@pasteur.fr; jean-marc.ghigo@pasteur.fr ${ }^{1}$ Département de Microbiologie, Unité de Génétique des Biofilms, Institut Pasteur, 25-28 rue du Dr. Roux, F-75015 Paris, France

Full list of author information is available at the end of the article
}

\section{Organism information}

Classification and features

Escherichia coli is a Gram-negative, rod-shaped, non-spore forming and facultative anaerobic species belonging to the Enterobacteriaceae family. They are commonly found in the intestines of endotherms and are taxonomically placed within the Gammaproteobacteria of the Proteobacteria phylum (Table 1).

E. coli 1094 is a commensal strain isolated from feces of a healthy human male. Like many other natural $E$. coli isolates, 1094 produces cellulose as the main component of its biofilm extracellular polymeric matrix (Fig. 1) [8], and has been used as a model for studying both transcriptional regulation and function of cellulose biosynthesis genes (bcs genes) [8, 9], as well as to analyze the structure of the cellulose secretion machinery [10]. Here, we investigate global genomic differences between representative members of $E$. coli phylogenetic group A and discuss their functional consequences on cellulose production.

E. coli 1094 was previously classified as a member of the phylogenetic group A based on triplex PCR with a combination of primers amplifying two genes (chuA and $y j a A)$, and an anonymous DNA fragment designated TSPE4.C2 [7]. Using all available complete genomes of phylogenetic group A, we performed a core genome 
Table 1 Classification and general features of Escherichia coli strain 1094

\begin{tabular}{|c|c|c|c|}
\hline MIGS ID & Property & Term & Evidence code $^{a}$ \\
\hline & Classification & Domain Bacteria & TAS [33] \\
\hline & & Phylum Proteobacteria & TAS [34] \\
\hline & & Class Gammaproteobacteria & TAS [34] \\
\hline & & Order "Enterobacteriales" & TAS $[34,35]$ \\
\hline & & Family Enterobacteraceae & TAS [36] \\
\hline & & Genus Escherichia & TAS $[37,38]$ \\
\hline & & Species Escherichia coli & TAS $[37,38]$ \\
\hline & & Type strain: 1094 & TAS \\
\hline & Gram stain & Negative & IDA, TAS [39] \\
\hline & Cell shape & Rod & IDA, TAS [39] \\
\hline & Motility & Motile & IDA, TAS [39] \\
\hline & Sporulation & Non & TAS [39] \\
\hline & Temperature range & $10^{\circ} \mathrm{C}-45^{\circ} \mathrm{C}$ & NAS \\
\hline & Optimum Temperature & $37^{\circ} \mathrm{C}$ & IDA \\
\hline & pH range; optimum & $5.5-8.0 ; 7$ & \\
\hline & Carbon source & Glucose & IDA \\
\hline MIGS-6 & Habitat & Human gut & \\
\hline MIGS-6,3 & Salinity & Not reported & \\
\hline MIGS-22 & Oxygen requirement & Facultative anaerobe & IDA, TAS $[39,40]$ \\
\hline MIGS-15 & Biotic relationship & Human specimen & NAS \\
\hline MIGS-14 & Pathogenicity & Nonpathogenic & NAS \\
\hline MIGS-4 & Geographic location & France & \\
\hline MIGS-5 & Sample collection & 1999 & \\
\hline MIGS-4,1 & Latitude & Not reported & \\
\hline MIGS-4,2 & Longitude & Not reported & \\
\hline MIGS-4,4 & Altitude & Not reported & \\
\hline
\end{tabular}

${ }^{\mathrm{a} E v i d e n c e ~ c o d e s ~-~ T A S ~ T r a c e a b l e ~ A u t h o r ~ S t a t e m e n t, ~ N A S ~ N o n-t r a c e a b l e ~ A u t h o r ~ S t a t e m e n t, ~ I D A ~ I n f e r r e d ~ f r o m ~ D i r e c t ~ A s s a y . ~ T h e s e ~ e v i d e n c e ~ c o d e s ~ a r e ~ f r o m ~ t h e ~ G e n e ~}$ Ontology project [12]

alignment to produce a phylogenetic tree, and found it to be in agreement with previous phylogenetic classification [11], showing that E. coli strain 1094 is closely related to strains ATCC 8739 and HS (Fig. 2).

\section{Genome sequencing information}

\section{Genome project history}

E. coli 1094 has been used as a model to study different aspects of cellulose biosynthesis and biofilm formation $[8,9]$. Cellulose production requires the expression of bacterial cellulose synthesis genes clustered in two divergent operons, as well as genes involved in general glucose metabolism [8], In order to further elucidate the genetic bases of cellulose synthesis, we chose to sequence E. coli 1094 using two approaches: Illumina and PacBio sequencing. While Illumina sequencing and subsequent downstream analysis generated 204 contigs, PacBio sequencing and assembly produced 4 contigs. A summary of the project information and its association with "Minimum Information about a Genome Sequence" [12] are provided in Table 2.

\section{Growth conditions and genomic DNA preparation}

E. coli 1094 was cultivated in LB medium overnight at $37{ }^{\circ} \mathrm{C}$. High quality genomic DNA for sequencing was extracted using the Wizard Genomic DNA Kit (Promega) (for Illlumina approach), or the QiaAMP DNA Mini Kit (QIAGEN) (for PacBio approach).

\section{Genome sequencing and assembly Illumina sequencing}

Whole genome library preparation (NEXTflex PCR-Free DNA-Seq kit, Bioo Scientific) and sequencing following standard protocols developed by the supplier were performed at the Genomics platform at the Pasteur Institute. Single reads averaging 110 base pairs were collected on a HiSeq2000 (Illumina, San Diego, CA). Approximately 8,285,636 reads were assembled using CLC Bio (version 4. 


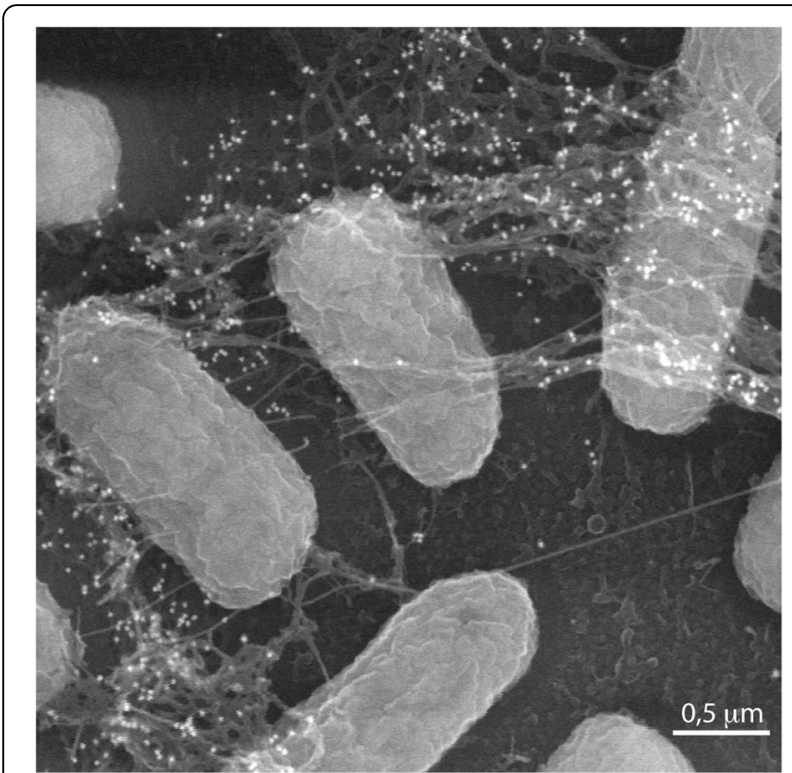

Fig. 1 Scanning electron microscopy of cellulose producing strain $E$. coli 1094. E. coli 1094. Bacteria with cellulose labeled by CBM29-1-2 and immunogold antibodies. Scanning electron microscopy and immunogold labelling were performed at LBCME facility, Faculte de Medecine de Tours, Tours, France)

20) giving a total of 204 contigs. The final Illumina-based sequence includes $4,982,209$ bases with a $\mathrm{G}+\mathrm{C}$ content of $50.81 \%$.

\section{PacBio sequencing}

Library preparation, sequencing, and assembly were performed by the Earlham Institute. PacBio sequencing libraries were prepared from $10 \mu \mathrm{g}$ DNA using standard Pacific Biosciences protocols (Pacific Biosciences, Menlo Park, CA). Following construction, libraries were size selected, from 7 to $50 \mathrm{~kb}$, using the Sage Science BluePippin ${ }^{\mathrm{Tm}}$ system with a $0.75 \%$ gel cassette. Libraries were run on the Pacific Biosciences RSII sequencer at 350pM using P6/C4 chemistry. A Single Molecule Real Time (SMRT) cell was used, yielding 150,292 reads, and 1213 megabases of sequence data. Reads were assembled using PacBio's hierarchical genome-assembly process (HGAP.3), with filtering and adaptor trimming performed as previously described [13]. The minimum seed read length used was $6 \mathrm{~kb}$, with a quality score cutoff of 0.8 . The Celera Assembler was used to produce 4 large contigs, using pre-assembled, errorcorrected reads. The maximum contig length produced was 4,903,991 bases.

\section{Illumina \& PacBio comparison}

Illumina single-end reads were mapped against the four large contigs generated by PacBio reads using the singleend mode of Bwa mem v0.7.4 [14] with default parameters (Fig. 3). Output SAM files were converted to BAM

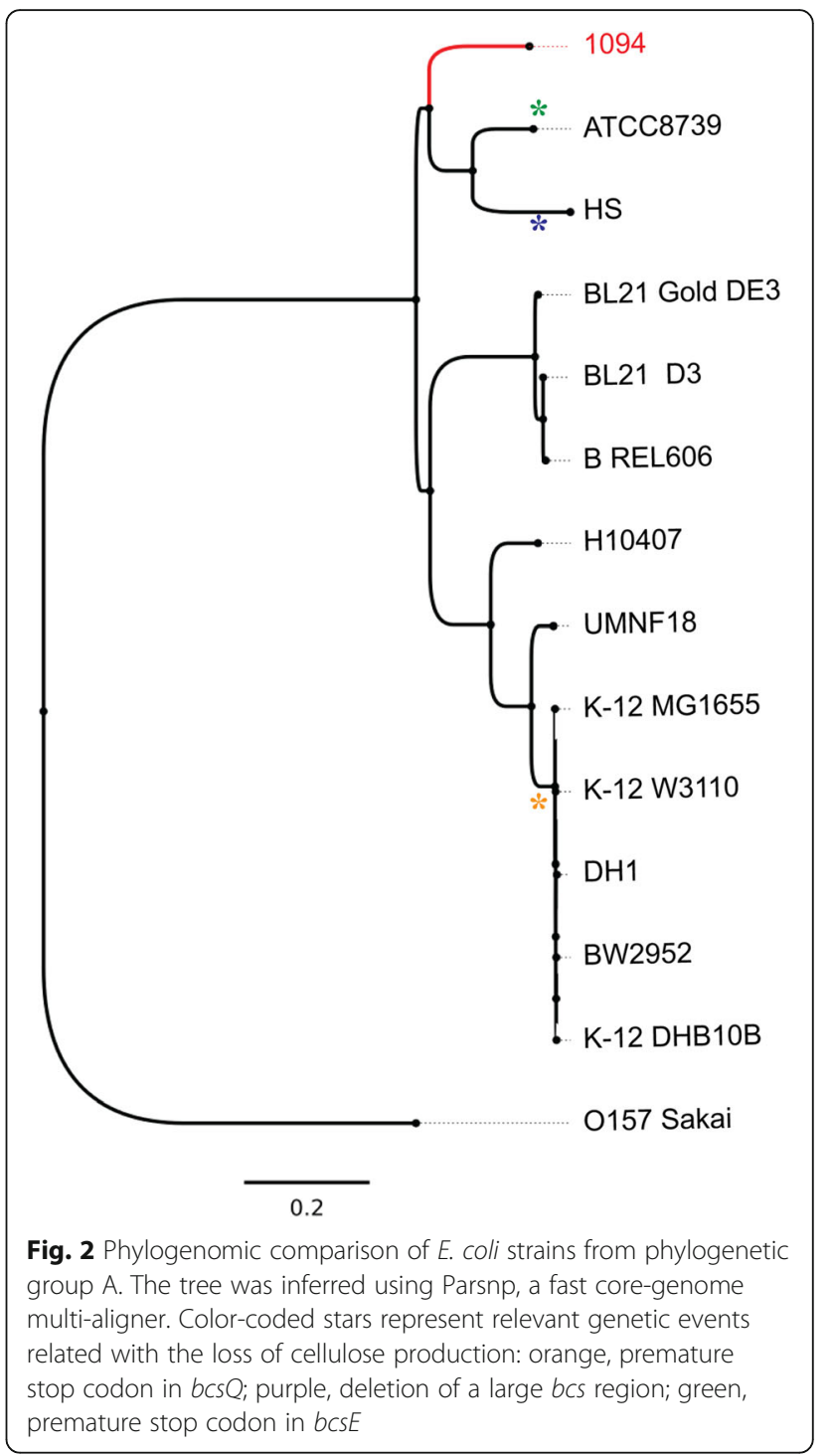

files using SAMtools v0.1.19 [15]. Sequencing coverage was computed using BEDtools v2.17.0 0 [16] and values were normalized to 1 (genomes are haploids), from the median coverage over the large contigs $(354 \times)$. The mapping coverage along the four PacBio contigs validated the sequence assemblies. Some peaks of high coverage are observed in unitig_0, unitig_1 and unitig_2 (Fig. 3a), which suggests that multiple copies of these regions exist. By contrast, the coverage of the complete unitig_3 indicates that there is likely more than one copy per chromosome within each cell.

SPAdes v 3.9.1 [17, 18] was used for assembling Illumina reads, for detecting putative plasmids sequence with the options '-plasmid,' '-k 21,51,71,' ccut-off auto' and '-careful'. Illumina reads were re-mapped onto the contigs using the single-end mode of Bwa mem v0.7.4 with default parameters. After converting output SAM files to BAM files by SAMtools, putative plasmid reads were extracted using 
Table 2 Project information

\begin{tabular}{lll}
\hline MIGS ID & Property & Term \\
\hline MIGS 31 & Finishing quality & High quality drafts \\
MIGS-28 & Libraries used & Two genomic libraries; one Illumina library, one PacBio standard library \\
MIGS 29 & Sequencing platforms & Illumina Miseq2000, Pacific Biosciences RSII \\
MIGS 31.2 & Fold coverage & $354 \times$ Illumina, 114.3× PacBio \\
MIGS 30 & Assemblers & CLC Bio (version 4.20), HGAP.3 \\
MIGS 32 & Gene calling method & GLIMMER2 \\
& Locus Tag & EC1094V2 \\
& Genbank ID & LT883139-LT883142 \\
& GenBank Date of Release & 05-DEC-2017 \\
& GOLD ID & Gp0267270 \\
& BIOPROJECT & PRJEB21000 \\
MIGS 13 & Source Material Identifier & CRBIP19.182 \\
& Project relevance & Human commensal \\
\hline
\end{tabular}

SAMtools (option 'view -F 4') and recorded in a Fastq file by picardtools 'SamToFastq' [19]. Putative plasmid reads were mapped against the four large PacBio contigs using the single-end mode of Bwa mem v0.7.4 with default parameters. The coverage computed by BEDtools (Fig. 3b) indicates that the complete unitig_3 is classified as a putative plasmid sequence and also appears to exist in high copy number. For unitig_0, unitig_1 and unitig_2, the mapping coverage shows some portions of large contigs classified as putative plasmids. This may correspond to plasmids with similar coverage to the chromosome, due to low copy number, or to misclassification by plasmidSPAdes.

\section{Sequence circularization}

PacBio scaffold sequences were compared against themselves with the bl2seq BLASTN algorithm [20], and ACT [21] was used for synteny visualization. The resulting overlapping sequences were easily identified between the beginning and the end of each large contigs, suggesting that all four PacBio large contigs are circular. To determine the size of the chromosome and each plasmid, the size of the overlapping region (Unitig_0: 15,155; Unitig_1: 8,919; Unitig_2: 13,971; Unitig_3: 9,380) was subtracted from the length of each contig (Unitig_0: 4,903,991; Unitig_1: 123,705; Unitig_ 2: 118,720; Unitig_3: 30,364); the final sizes are reported in Table 3.

\section{Genome annotation}

The complete genome sequence was annotated with the RAST server [22] which predicted 5028 coding sequences and 110 RNAs.

\section{Genome properties}

A summary of the genome of E. coli 1094 is included in Table 3. The genome statistics are provided in Table 4 .
Three putative plasmids were identified, and found to be circular. The genome of strain 1094 has a total length of $5,176,780$ base pairs and a $\mathrm{G}+\mathrm{C}$ content of $50.9 \%$. The majority of the protein-coding genes were assigned a putative function (78.8\%) while the remaining ones were annotated as hypothetical proteins. Genes in internal clusters were detected using BLASTclust with thresholds of $70 \%$ covered length and 30\% sequence identity [23]. CRISPR, transmembrane helice, signalP and Pfam protein families predictions were done using CRISPRFinder [24], TMHMM Server v.2.0 [25], SignalP 4.0 [26] and Pfam 29.0 [27], respectively. The distribution of genes into COGs functional categories is presented in Table 5 .

\section{Insights from the genome sequence}

E. coli 1094 sequence was aligned against selected genomes belonging to $E$. coliphylogenetic group A. Alignment and posterior NJ (neighbor joining) phylogenetic reconstruction was carried out with Parsnp, a fast core-genome multialigner, using default parameters [28]. Alignment and tree visualization was done with Gingr, a dynamic visual platform (Fig. 2) [28].

We selected five $E$. coli genomes that are representative of the multiple clades within phylogenetic group $\mathrm{A}$, and performed orthologous clustering to examine the genomic differences of E. coli 1094. [E. coli K12 W3110 (accession $\mathrm{n}^{\circ}$ : AP009048), E. coli BL21-Gold(DE3)pLysS AG' (accession CP001665), E. coli HS (accession $n^{\circ}$ : CP000802), E. coli ATCC 8739 (accession $n^{\circ}$ : CP000946) and the prototypical enterotoxigenic strain of E. coli ETEC H10407 (accession FN649414)] (Fig. 4). Our analysis reveals a core genome of 3409 genes shared among all strains (Fig. 4). E. coli ETEC H10407 possesses the highest number of specific genes (834), followed by E. coli 1094 (499), E. coli 


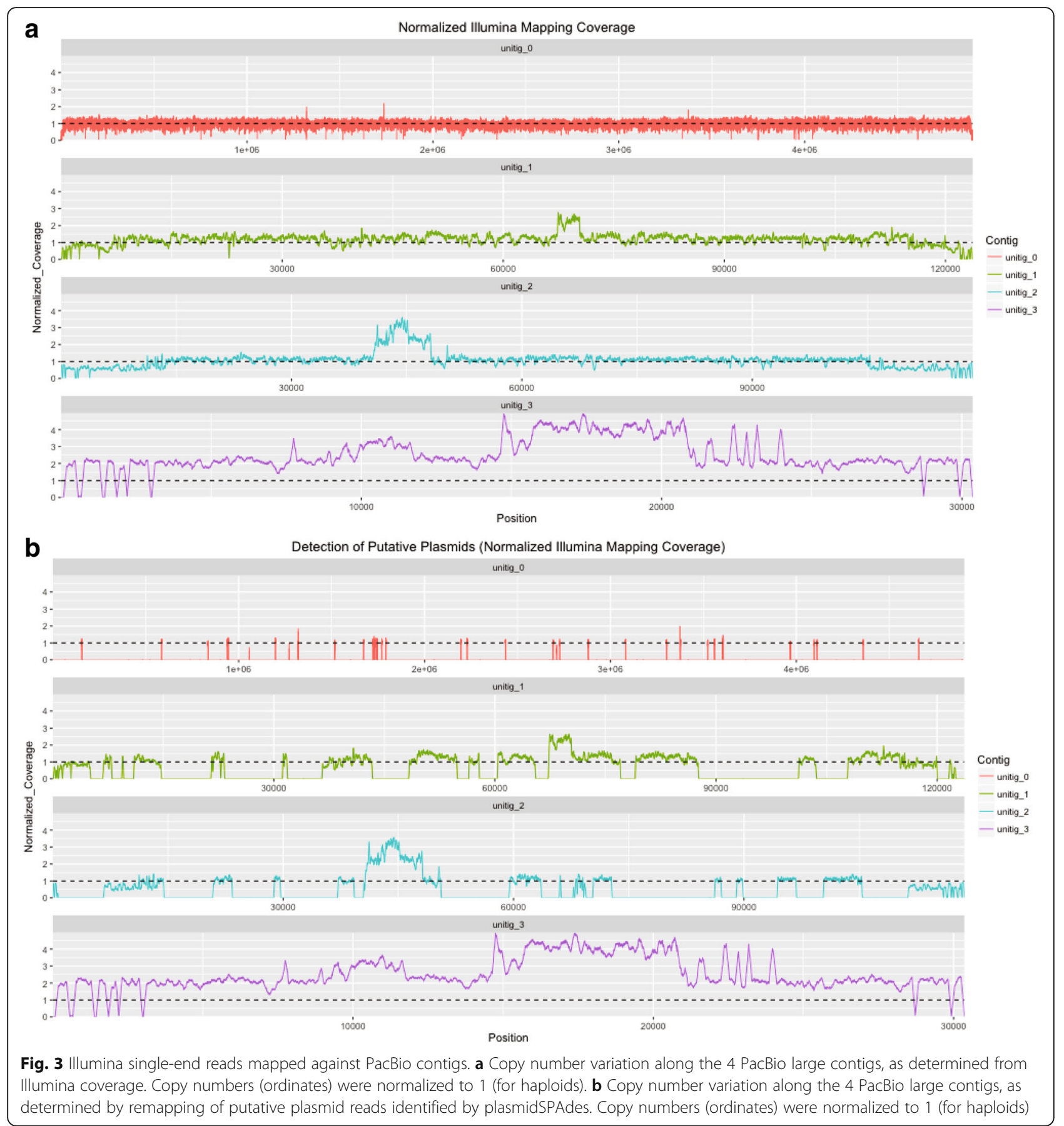

Table 3 Summary of 1094 genome: one chromosome and 3 putative plasmids

\begin{tabular}{lllll}
\hline Label & Size $(\mathrm{Mb})$ & Topology & INSDC identifier & RefSeq ID \\
\hline Chromosome & 4.888 & Circular & LT883139 & \\
Plasmid 1 & 0.115 & Circular & LT883140 & \\
Plasmid 2 & 0.105 & Circular & LT883141 & \\
Plasmid 3 & 0.021 & Circular & LT883142 & \\
\hline
\end{tabular}

HS (440), E. coli BL21-Gold(DE3)pLysS AG' (292), E. coli K12 W3110 (241) and E. coli ATCC8739 (216).

The analysis of the location of genes only present in $E$. coli 1094 identified eight 1094-specific regions, most of which are prophage or phage-associated proteins; two regions represent a putative complete prophage, containing genes encoding the phage capsid tail and replication proteins (Fig. 4). Other 1094-specific regions of interest contain CRISPR associated proteins. We also identified clusters only present in some, but not all E. coli strains 
Table 4 Genome statistics

\begin{tabular}{lll}
\hline Attribute & Value & \% of Total \\
\hline Genome size (bp) & $5,176,780$ & 100.0 \\
DNA coding (bp) & $4,503,495$ & 86.9 \\
DNA G + C (bp) & $2,340,599$ & 50.9 \\
DNA scaffolds & 4 & 100.0 \\
Total genes & 5138 & 100.0 \\
Protein coding genes & 5028 & 97.8 \\
RNA genes & 110 & 2.1 \\
Pseudo genes & 0 & 0 \\
Genes in internal clusters & 232 & 4.5 \\
Genes with function prediction & 4390 & 85.4 \\
Genes assigned to COGs & 4827 & 93.9 \\
Genes with Pfam domains & 4377 & 85.2 \\
Genes with signal peptides & 398 & 7.7 \\
Genes with transmembrane helices & 1099 & 21.4 \\
CRISPR repeats & 11 & 0.2 \\
\hline
\end{tabular}

analyzed, including (i) a putative complete type VI secretion system (present in 1094 and HS), (ii) a type III secretion system cluster (absent in BL21-Gold(DE3) and K12 W3110 strains of E. coli) (iii) a cluster of genes encoding invasins and an iron acquisition system, (present in 1094 and H10407), and (iv) several cellulose biosynthesis genes present in all strains, except for E. coli HS.

We performed a basic BLAST (BLASTN 2.6.1+, [29]) of each smaller contig identified, and report the following results. Unitig_1 shows sequence homology to Salmonella enterica subsp. enterica serovar Senftenberg strain 775 W plasmid pSSE-ATCC-43845 (Accession: CP016838.1), with $33 \%$ of the contig showing $99 \%$ identity. Unitig_2 displays sequence homology to Klebsiella pneumoniae subsp. pneumoniae strain 234-12 plasmid pKpn23412-362, with 39\% of the contig showing 99\% identity (Accession: CP011314. 1 ); other regions of the contig show homology to multiple E. coli strains and plasmids. Unitig_3 displays sequence homology to Citrobacter freundii strain B38 plasmid pOZ182; 68\% of the contig shows 96\% identity (Accession: CP016765.1); in addition, two separate regions of 7.5 and 2. $1 \mathrm{~kb}$ are highly homologous to E. coli plasmid pV004-a DNA and pV001-a DNA (Accession: LC056140.1 and

Table 5 Number of genes associated with general COG functional categories

\begin{tabular}{|c|c|c|c|}
\hline Code & Value & \%age & Description \\
\hline J & 179 & 3.5 & Translation, ribosomal structure and biogenesis \\
\hline A & 3 & 0.05 & RNA processing and modification \\
\hline K & 315 & 6.3 & Transcription \\
\hline L & 254 & 5.05 & Replication, recombination and repair \\
\hline B & 0 & 0 & Chromatin structure and dynamics \\
\hline D & 45 & 0.9 & Cell cycle control, Cell division, chromosome partitioning \\
\hline V & 58 & 1.15 & Defense mechanisms \\
\hline $\mathrm{T}$ & 159 & 3.16 & Signal transduction mechanisms \\
\hline M & 285 & 5.66 & Cell wall/membrane biogenesis \\
\hline N & 62 & 1.23 & Cell motility \\
\hline U & 116 & 2.3 & Intracellular trafficking and secretion \\
\hline O & 172 & 3.42 & Posttranslational modification, protein turnover, chaperones \\
\hline C & 308 & 6.12 & Energy production and conversion \\
\hline G & 771 & 15.3 & Carbohydrate transport and metabolism \\
\hline $\mathrm{E}$ & 318 & 6.32 & Amino acid transport and metabolism \\
\hline $\mathrm{F}$ & 109 & 2.16 & Nucleotide transport and metabolism \\
\hline H & 136 & 2.7 & Coenzyme transport and metabolism \\
\hline । & 139 & 2.76 & Lipid transport and metabolism \\
\hline P & 280 & 5.56 & Inorganic ion transport and metabolism \\
\hline Q & 67 & 1.33 & Secondary metabolites biosynthesis, transport and catabolism \\
\hline $\mathrm{R}$ & - & - & General function prediction only \\
\hline S & 1051 & 20.9 & Function unknown \\
\hline- & - & - & Not in COGs \\
\hline
\end{tabular}




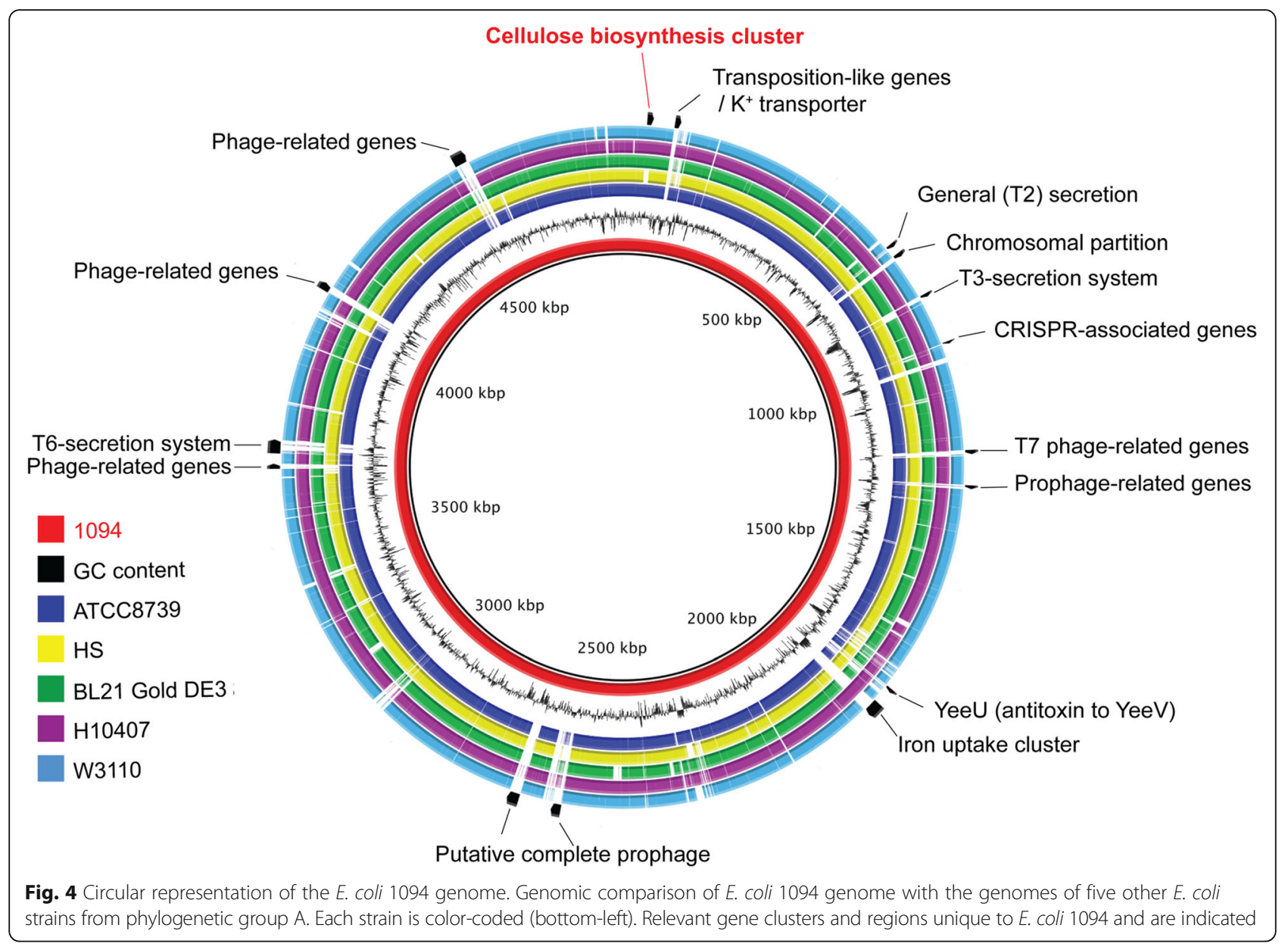

LC056078.1). Taken together, this suggests that 1094 expresses 3 distinct circular plasmids.

\section{Comparative analysis of the bcs (bacterial cellulose synthesis) region}

We compared the region corresponding to the E. coli $1094 b c s$ operon with corresponding regions in the strains E. coli W3110, HS, BL21-Gold(DE3)pLysS AG', ATCC 8739, and H10407, which are representative of phylogenetic group A. This analysis shows that whereas only partial fragments of the external genes of the $b c s$ operon ( $b c s C$ and $b c s G$ ) exist in E. coli HS, the five other strains analyzed contain all genes within the $b c s$ operon (Fig. 5). K12 derivative strains do not produce cellulose, as they contain a premature stop codon in the gene $b c s Q$, due to a single nucleotide polymorphism (SNP) in the region TTG/TAG (17 T > A) (Fig. 5) [30]. Serra et al., repaired this SNP, which resulted in cellulose production in E. coli K12 W3110, suggesting that the premature stop codon in $b c s \mathrm{Q}$ could either affect the function of $\mathrm{BcsQ}$, or has a polar effect on neighboring genes [30]. The other SNPs (relative to E. coli 1094) observed in the bcs operons of E. coli K-12 strains result either in synonymous codons or in conservative amino acid exchanges. By contrast, comparison of the bcs operon sequence between E. coli 1094 and E. coli ATCC8739 revealed 100\% sequence identity between $b c s Q, b c s R, b c s A$ and $b c s B$ genes. Finally, the sequence of the $b c s$ operon in E. coli ATCC8739 contains one SNP in $b c s E$ that leads to a premature stop codon, and may also have a polar effect on $b c s F$ and $b c s G$, which are essential for cellulose production [8] (Fig. 5). E. coli strains BL21-Gold(DE3)pLysS AG' and H10407 contain multiple SNPs in bcs genes that are essential for cellulose biosynthesis. Some of these SNPs lead to amino acid changes that could negatively affect the function of these proteins. $b c s A$ is particularly interesting, as a pairwise genetic comparison in all six $E$. coli strains analyzed revealed the existence of multiple $b c s A$ SNPs (between 21 and 29, depending on the strain pair compared). None of the SNPs resulted in amino acid exchange or a premature stop codon, suggesting a strong selection pressure for this specific amino acid composition. Our phylogenetic analysis of the 


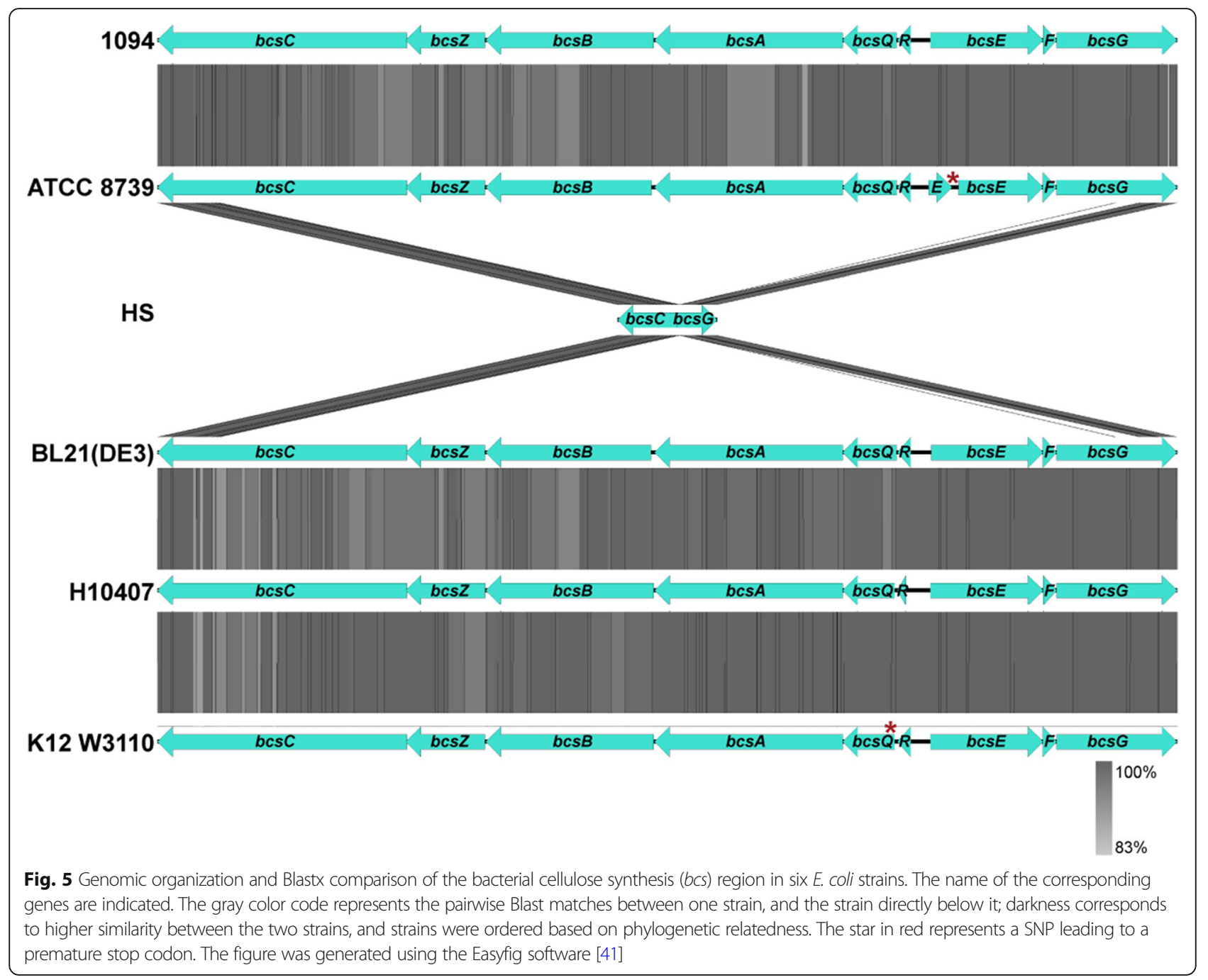

phylogenetic group A allowed us to localize these evolutionary events, by following a parsimony criterion, which allowed us to infer that mutations leading to premature stop codons took place in two separate evolutionary events: the branch of the strain ATCC8739 and in the K12 common ancestor (Fig. 2). These mutations resulted in sequence interruption of $b c s E$ and $b c s Q$ respectively.

\section{Cellulose production of $E$. coli strains}

Cellulose production was tested in the six E. coli strains analyzed in this study. We monitored colony fluorescence on LB-calcofluor plates, which is a common assay used to detect cellulose biosynthesis [31] (Fig. 6). This analysis revealed that only E. coli 1094 strain produced detectable levels of cellulose. As was previously shown, laboratory E. coli $\mathrm{K} 12$ strains do not produce cellulose

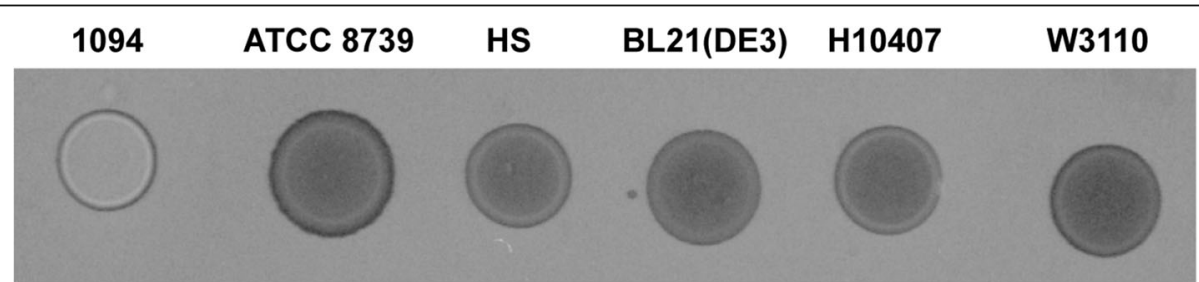

Fig. 6 Cellulose production in six E. coli strains. Cellulose secretion was evaluated by the binding of calcofluor (CF), as visualized by colony fluorescence under ultraviolet light (fluorescence appears white in image). Five microliters of an overnight culture was spotted onto LB agar plates, containing $0.02 \%$ of CF and incubated for $24 \mathrm{~h}$ at $30^{\circ} \mathrm{C}$. Images were acquired using a G:BOX imaging system (Syngene, Frederick, MD) 
[30]. Here we show that, as expected, E. coli HS is not able to produce this polymer, as it was known to lack all bcs genes. Interestingly, cellulose production was not detected in E. coli ATCC 8739, suggesting that the SNP in the region TGT/TGA (303 T $>\mathrm{A})$ of $b c s E$ described above could have a polar effect on the downstream genes. While the bcsEFG operon is essential for cellulose production [8], bcsE is only necessary for maximal cellulose production [32], suggesting that the inability to synthetize detectable levels of cellulose may be due to a polar effect of the bcsEFG operon. Finally, cellulose production was not detected in strains BL21-Gold(DE3) pLysS AG' and H10407, which could be explained by the presence of multiple amino acid changes that potentially affect the biological activity of BcsB, BcsC and BcsQ proteins, which are essential for cellulose production.

\section{Conclusions}

The human commensal intestinal isolate Escherichia coli strain 1094 naturally produces cellulose, a polysaccharide known to contribute to adhesion and biofilm development. We compared genomic content and cellulose production of closely related $E$. coli strains in phylogenetic group A, and found 1094 to be the sole strain to produce measureable levels of cellulose, and conclude that these strains lack the capacity to produce cellulose due to one or several SNPs in cellulose biosynthesis genes: non-synonymous SNPs in $b c s B, b c s C, b c s Q$, and a nonsense mutation in $b c s E$. The genome sequencing and annotation here provides valuable information for future study of the regulation of the $b c s$ genes and cellulose production.

\section{Abbreviations}

Bcs: Bacterial cellulose synthesis; CF: Calcofluor; RAST: Rapid Annotation using Subsystem Technology; SNPs: Single nucleotide polymorphism

\section{Acknowledgements}

E. coli strain 1094 was a generous gift from Chantal Le Bouguennec. We would like to thank Magali Tichit for his technical help and Evelyne Begaud and the CRBIP (Centre de Ressources Biologiques de l'Institut Pasteur) for providing the E. coli ATCC8739 strain. High-throughput sequencing was performed by the Genomics Platform of the Institut Pasteur, a member of the France Génomique consortium [ANR10-INBS-09-08]

\section{Funding}

This work was supported by the Institut Pasteur and grants from the French Government's Investissement d'Avenir program, Laboratoire d'Excellence: Integrative Biology of Emerging Infectious Diseases [grant nANR-10-LABX62-IBEID], and from the Fondation pour la Recherche Médicale grant, [Equipe FRM DEQ20140329508].

\section{Authors' contributions}

$J B$ and JMG conceived the project. JB and LGV conducted phylogenomic studies. JB, LGV, AKW and JMG wrote the manuscript. JB and AKW performed the laboratory experiments. CB performed Illumina library preparations, and Illumina sequencing runs. JB and LGV assembled and annotated the genome. VK performed comparative analysis of Illumina and PacBio sequencing, and identified plasmids. All authors read and approved the final version of the manuscript.

\section{Competing interests}

The authors declare that they have no competing interests.

\section{Publisher's Note}

Springer Nature remains neutral with regard to jurisdictional claims in published maps and institutional affiliations.

\section{Author details}

${ }^{1}$ Département de Microbiologie, Unité de Génétique des Biofilms, Institut Pasteur, 25-28 rue du Dr. Roux, F-75015 Paris, France. Département de Génomes et Génétique, Unité de Biologie des Bactéries Intracellulaires, Institut Pasteur, 25-28 rue du Dr. Roux, F-75015 Paris, France. ${ }^{3}$ Centre National de la Recherche Scientifique (CNRS). UMR 3525, 75724 Paris, France. ${ }^{4}$ Institut Pasteur - Hub Bioinformatique et Biostatistique - C3BI, USR 3756 IP CNRS, Paris, France. ${ }^{5}$ Institut Pasteur, Plate-forme Génomique, Pôle Biomics, CITECH 25-28 rue du Dr. Roux, F-75015 Paris, France.

Received: 21 August 2017 Accepted: 13 April 2018

Published online: 09 May 2018

\section{References}

1. Stoodley P, Sauer K, Davies DG, Costerton JW. Biofilms as complex differentiated communities. Annu Rev Microbiol. 2002;56:187-209. Available from: http://www.ncbi.n/m.nih.gov/pubmed/12142477. Cited 17 Oct 2016.

2. Flemming H-C, Wingender J. The biofilm matrix. Nat Rev Microbiol. 2010;8: 623-33. Available from: http://www.ncbi.nlm.nih.gov/pubmed/20676145. Cited 17 Oct 2016

3. Delmer DP, Amor Y. Cellulose biosynthesis. Plant Cell. 1995;7:987-1000. Available from: http://www.ncbi.nlm.nih.gov/pubmed/7640530. Cited 6 Oct 2016.

4. Römling U. Molecular biology of cellulose production in bacteria. Res Microbiol. 2002;153:205-12. Available from: http://www.ncbi.nlm.nih.gov/ pubmed/12066891. Cited 17 Oct 2016.

5. Römling U, Michael Y. Galperin. HHS Public Access. 2016;23:545-57.

6. Hilali F, Ruimy R, Saulnier P, Barnabé C, Lebouguénec C, Tibayrenc M, et al. Prevalence of virulence genes and clonality in Escherichia coli strains that cause bacteremia in cancer patients. Infect Immun. 2000;68: 3983-9. Available from: http://www.ncbi.n/m.nih.gov/pubmed/10858212. Cited 6 Oct 2016

7. Clermont O, Bonacorsi S, Bingen E. Rapid and simple determination of the Escherichia coli phylogenetic group. Appl Environ Microbiol. 2000; 66:4555-8. Available from: http://www.ncbi.nlm.nih.gov/pubmed/ 11010916. Cited 6 Oct 2016.

8. Da Re S, Ghigo J-M. A CsgD-independent pathway for cellulose production and biofilm formation in Escherichia coli. J Bacteriol. 2006; 188:3073-87. Available from: http://www.ncbi.nlm.nih.gov/pubmed/ 16585767. Cited 6 Oct 2016.

9. Le Quéré B, Ghigo J-M. BcsQ is an essential component of the Escherichia coli cellulose biosynthesis apparatus that localizes at the bacterial cell pole. Mol Microbiol. 2009;72:724-40. Available from: http://www.ncbi.nlm.nih.gov/ pubmed/19400787. Cited 6 Oct 2016

10. Krasteva PV, Bernal-Bayard J, Travier L, Martin FA, Kaminski P-A, Karimova G, Fronzes R, Ghigo JM. Insights into the structure and assembly of a bacterial cellulose secretion system. Nat Commun. 2017. Available from: https://www. nature.com/articles/s41467-017-01523-2\#Sec24. Cited 12 Dec 2017.

11. Skippington $E$, Ragan MA. Phylogeny rather than ecology or lifestyle biases the construction of Escherichia coli-Shigella genetic exchange communities. Open Biol. 2012;2:120112. Available from: http://www.ncbi.nlm.nih.gov/ pubmed/23091700. Cited 16 Jan 2017.

12. Field D, Garrity G, Gray T, Morrison N, Selengut J, Sterk $P$, et al. The minimum information about a genome sequence (MIGS) specification. Nat Biotechnol. 2008;26:541-7. Available from: https://doi.org/10.1038/nbt1360.

13. Chin C-S, Alexander DH, Marks P, Klammer AA, Drake J, Heiner C, et al. Nonhybrid, finished microbial genome assemblies from long-read SMRT sequencing data. Nat Methods. 2013;10:563-9. Available from: http://www. nature.com/doifinder/10.1038/nmeth.2474. Cited 20 Jan 2017.

14. Li H, Durbin R. Fast and accurate short read alignment with burrowswheeler transform. Bioinformatics. 2009;25:1754-60.

15. Li H, Handsaker B, Wysoker A, Fennell T, Ruan J, Homer N, et al. The sequence alignment/map format and SAMtools. Bioinformatics. 2009;25:2078-9.

16. Quinlan AR, Hall IM. BEDTools: a flexible suite of utilities for comparing genomic features. Bioinformatics. 2010;26:841-2. 
17. Nurk S, Bankevich A, Antipov D, Gurevich AA, Korobeynikov A, Lapidus A, et al. Assembling single-cell genomes and mini-metagenomes from chimeric MDA products. J Comput Biol. 2013;20:714-37. Available from: http://www. pubmedcentral.nih.gov/articlerender.fcgi?artid=3791033\&tool= pmcentrez\&rendertype $=$ abstract.

18. Antipov D, Hartwick N, Shen M, Raiko M, Pevzner PA. plasmidSPAdes: assembling plasmids from whole. BioRxiv; 2016. p. 2014-5.

19. Picard Tools by Broad Institute GitHub Pages. Available from: http:// broadinstitute.github.io/picard/. Cited 31 Aug 2016.

20. Tatusova TA, Madden TL. BLAST 2 SEQUENCES, a new tool for comparing protein and nucleotide sequences. FEMS Microbiol Lett. 1999;174:247-50.

21. Carver TJ, Rutherford KM, Berriman M, Rajandream MA, Barrell BG, Parkhill J. ACT: the Artemis comparison tool. Bioinformatics. 2005;21:3422-3.

22. Aziz RK, Bartels D, Best AA, DeJongh M, Disz T, Edwards RA, et al. The RAST server: rapid annotations using subsystems technology. BMC Genomics. 2008;9:75. Available from: http://www.ncbi.nlm.nih.gov/pubmed/18261238. Cited 6 Oct 2016.

23. BLASTclust [https://www.ncbi.nlm.nih.gov/pmc/articles/PMC4987908/]. Accessed 6 Nov 2015.

24. Grissa I, Vergnaud G, Pourcel C. CRISPRcompar: a website to compare clustered regularly interspaced short palindromic repeats. Nucleic Acids Res. 2008:36:W145.

25. Krogh a, Larsson B, von Heijne G, Sonnhammer EL. Predicting transmembrane protein topology with a hidden Markov model: application to complete genomes. J Mol Biol. 2001;305:567-80.

26. Petersen TN, Brunak S, Von Heijne G, Nielsen H. SignalP 4.0: discriminating signal peptides from transmembrane regions. Nat Methods. 2011;8:785-6.

27. Finn RD, Coggill P, Eberhardt RY, Eddy SR, Mistry J, Mitchell AL, et al. The Pfam protein families database: towards a more sustainable future. Nucleic Acids Res. 2016;44:D279-85.

28. Treangen TJ, Ondov BD, Koren S, Phillippy AM. The harvest suite for rapid core-genome alignment and visualization of thousands of intraspecific microbial genomes. Genome Biol. 2014;15:524. Available from: http://www. ncbi.nlm.nih.gov/pubmed/25410596. Cited 22 Nov 2016.

29. Zhang Z, Schwartz S, Wagner L, Miller W. A greedy algorithm for aligning DNA sequences. J Comput Biol. 2000;7:203-14.

30. Serra DO, Richter AM, Hengge R. Cellulose as an architectural element in spatially structured escherichia coli biofilms. J Bacteriol. 2013;195:5540-54. Available from: http://www.ncbi.nlm.nih.gov/pubmed/24097954. Cited 6 Oct 2016.

31. Leigh JA, Signer ER, Walker GC. Exopolysaccharide-deficient mutants of rhizobium meliloti that form ineffective nodules. Proc Natl Acad Sci. 1985;82: 6231-5. Available from: http://www.pnas.org/cgi/doi/10.1073/pnas.82.18.6231.

32. Fang X, Ahmad I, Blanka A, Schottkowski M, Cimdins A, Galperin MY, et al. GIL, a new c-di-GMP-binding protein domain involved in regulation of cellulose synthesis in enterobacteria. Mol Microbiol. 2014;93:439-52.

33. Woese CR, Kandlert O, Wheelis ML. Towards a natural system of organisms: proposal for the domains archaea, Bacteria, and Eucarya. Evolution (N Y). 1990;87:4576-9.

34. Garrity GM, Lilburn T. Volume 2: the Proteobacteria, part B: the Gammaproteobacteria. In: Garrity G, Brenner DJ, Krieg NR, Staley JR, editors. Bergey's man. Syst. Bacteriol; 2005.

35. Williams KP, Kelly DP. Proposal for a new class within the phylum Proteobacteria, Acidithiobacillia classis nov., with the type order Acidithiobacillales, and emended description of the class Gammaproteobacteria. Int J Syst Evol Microbiol. 2013;63:2901-6.

36. Rahn RE, Ewing WH, Farmer JJ, Brenner DONJ. Proposal of Enterobacteriaceae fam. nov., norn. rev. to replace Enterobacteriaceae Rahn 1937, nom. fam. cons. (Opin. 15, Jud. Comm. 1958), which lost standing in nomenclature on 1 January 1980; 1980. p. 674-5.

37. Escherich T. Die Darmbakterien des Säuglings und ihre Beziehungen zur Physiologie der Verdauung; 1886. p. 63-74.

38. Editorial Board (for the Judicial Commission of the International Committee on Bacteriological Nomenclature). Bacterial Nomenclature and Taxonomy. Int J Syst Bacteriol. 1959;35-6.

39. Welch RA. The genus Escherichia. Prokaryotes prokaryotic biol. Symbiotic Assoc; 2006. p. 60-71. Available from: http://www.springer.com/life +sciences/microbiology/book/978-3-642-30193-3.

40. Scheutz F, Strockbine NA. Genus I. Escherichia Castellani and Chalmers 1919, 941T. Bergey's Man Syst Bacteriol. 2005;2:607-24.

41. Sullivan MJ, Petty NK, Beatson SA. Easyfig: a genome comparison visualizer. Bioinformatics. 2011;27:1009-10. Available from: http://www.ncbi.nlm.nih. gov/pubmed/21278367. Cited 11 Jan 2017.

\section{Ready to submit your research? Choose BMC and benefit from:}

- fast, convenient online submission

- thorough peer review by experienced researchers in your field

- rapid publication on acceptance

- support for research data, including large and complex data types

- gold Open Access which fosters wider collaboration and increased citations

- maximum visibility for your research: over $100 \mathrm{M}$ website views per year

At BMC, research is always in progress.

Learn more biomedcentral.com/submissions 